\title{
A Systematic Study on Au-Capped Si Nanowhiskers for Size-Dependent Improved Biosensing Applications
}

\author{
İsa Şeker ${ }^{1} \cdot$ Ali Karatutlu ${ }^{2}$ (I) $\cdot$ Kurtuluş Gölcük ${ }^{3} \cdot$ Mehmet Karakız $^{4} \cdot$ Bülend Ortaç $^{2}$
}

Received: 26 February 2020 / Accepted: 26 May 2020 / Published online: 2 June 2020

(C) Springer Science+Business Media, LLC, part of Springer Nature 2020

\begin{abstract}
Reducing the distance between the fluorescence molecules and noble metal (resonant) nanostructures is known to advance the process of electromagnetic coupling and energy transfer, which in return yields fluorescence enhancement particularly exploited for improved biomedical applications. In this study, Au-capped Si nanowhiskers (NWs) at various sizes were fabricated using a vapor-liquid-solid (VLS) mechanism for systematically investigating the dependence of the size of the Au-capped Si NWs on the fluorescence enhancement factor with respect to the fluorescence emission from Rhodamine 6G (Rh-6G) fluorophore. Opposite to what is anticipated from the literature, the maximum enhancement was obtained for the sample for which the Aunanoparticle (NP) capping is well isolated from the fluorophore and the vertical distance between the fluorophore and the plasmonic metal nanoparticle is largest. Numerical simulations using the finite element method (FEM) were shown to support the experimental optical response results. Four-point probe I-V measurements also show that the Schottky ideality factor of Aucapped Si NWs decays exponentially upon the rise in the fluorescence enhancement factor.
\end{abstract}

Keywords Si nanowhiskers $\cdot$ Plasmon $\cdot$ Fluorescence enhancement $\cdot$ Biomedical

\section{Introduction}

Spontaneous emission from fluorescence molecules can be improved by coupling a resonant cavity [1]. For such purposes, semiconductor nanowires including silicon (Si) nanowires are considered to be one of the promising structures

Electronic supplementary material The online version of this article (https://doi.org/10.1007/s11468-020-01195-7) contains supplementary material, which is available to authorized users.

\section{İsa Şeker \\ isaseker05@gmail.com \\ $\triangle$ Ali Karatutlu \\ ali@unam.bilkent.edu.tr}

1 Alyse Built-in Appliances, Organized Industrial Zone, 4th District, 05300 Merzifon, Amasya, Turkey

2 UNAM - National Nanotechnology Research Center and Institute of Materials Science and Nanotechnology, Bilkent University, 06800 Ankara, Turkey

3 Institute of Experimental Epileptology and Cognition Research, Life and Brain Center, University of Bonn Medical Center, Sigmund-Freud Str. 25, 53127 Bonn, Germany

4 Department of Mechatronics Engineering, Cumhuriyet University, 58140 Sivas, Turkey which are exploited due to their two dimensional (2D) quantum confinement effect (QCE) [2]. This effect alters their optical properties significantly in combination with the associated geometrical structure. In recent years, a group of such structures known as nanowhiskers (NWs) has been developed for different applications including nanoelectronic, optoelectronic, and photovoltaic devices along with chemical and biosensors [3, 4]. Furthermore, surface plasmons can be generated when NWs are capped with noble metal nanostructures. These surface plasmons lead to enhancement of the local electromagnetic field due to the relatively high extinction of the light at plasmonic extinction wavelengths [3]. When NWs are sufficiently close to one another where an analyte (or a fluorophore) is located, a relatively large Raman signal can be obtained [5]. Gold ( $\mathrm{Au}$ ) NPs are one of the most common plasmonic nanoparticles owing to their size-dependent optical properties [6]. On the other hand, both excitation and emission processes can be enhanced upon placing a fluorophore nearby these nanoparticles [1]. In other words, comparatively, a higher excitation and the radiative decay rates of the fluorophores cause enhancement of the fluorescence emission. It was also suggested that the fluorescence enhancement can be improved further when the plasmonic extinction wavelengths overlap with the emission wavelength of the fluorophores [7]. Overall, it is considered that the geometrical 
parameters of Au NPs including shape, size, and the distance between the fluorophore and metal nanoparticle play a significant role in the fluorescence enhancement [1]. Different strategies were engaged in the growth of Si NWs including vaporliquid-solid (VLS) [8-10], solid-liquid-solid [11, 12], epitaxial (vapor-solid-solid) [13, 14], oxide-assisted VLS [15], and other interesting routes [2, 16-18]. Nevertheless, the VLS is the most widely preferred growth method catalyzed by metal (Au)-Si alloy where fabrication of NWs can be conducted with the predefined diameter, length, and position of NWs on the substrate [4]. Furthermore, Si NW cone shape also results in an enhanced extinction of the exposed light over a range of wavelengths and angles of incidence [19]. Despite the remarkable studies mentioned so far (and references therein), to the authors' knowledge, the fluorescence enhancement of a fluorophore was not studied systematically depending on the geometrical parameters of Si NWs with the controlled morphology, diameter, and length of Si NWs. To the best of our knowledge, we report the first systematic study of Aucapped Si NWs to be utilized for the fluorescence enhancement of Rhodamine $6 \mathrm{G}(\mathrm{Rh}-6 \mathrm{G})$ based on the geometrical variations of the Si NWs. These geometrical variations were found to affect the extinction of the incident light through $\mathrm{Au}-$ capped Si NWs and thus reveal the optimum cone size for the fluorescence enhancement of the Rh-6G. The theoretical results obtained using the finite element method (FEM) are validated using the experimental optical responses of Au-capped Si NWs with different Si NWs geometry. The structural, elemental, and electrical properties of the Au-capped Si NWs were also correlated with the optical results.

\section{Experimental Section}

\section{Numerical Simulations}

Numerical simulations were performed using the FEM for all samples by commercial software Comsol 5.0. Three different geometrical models were formed for one NW and three NWs separately for S-1, S-2, and S-3 using the geometry tab almost matching with the mean sizes given in Table 1 . The dielectric functions for $\mathrm{Au}$ and $\mathrm{Si}$ were interpolated from the previous studies $[20,21]$. The simulations were operated for the wavelength range of $532-647 \mathrm{~nm}$ with a step of $5 \mathrm{~nm}$.

\section{Fabrication of Au-Capped Si NWs}

Au-capped Si NWs were grown on top of high purity n-type $\mathrm{Si}$ (111) wafer substrates with a size of $10 \mathrm{~mm}^{2}$ by the VLS technique using electron beam deposition (EBD) system as one of the authors previously reported [3]. Before the deposition process, the substrates were cleaned in accordance with the Radio Corporation of America (RCA) procedures, which include the standard clean-1, 2 (SC-1,2) and diluted hydrofluoric acid (DHF) steps. After rinsing and blowdrying with an $\mathrm{N}_{2}$ gun, they were immediately transferred into the main chamber of the EBD system. As the first step of the growth process, 2.2-nm thick Au layers were grown on top of the substrates at room temperature. Following this, the samples were annealed at $750{ }^{\circ} \mathrm{C}$ for $40 \mathrm{~min}$ to form Au nanodroplets. Finally, Si NW growth was carried out at $700{ }^{\circ} \mathrm{C}$ substrate temperature with a growth rate of $1.0 \AA / \mathrm{s}$ and deposition times of $120 \mathrm{~min}, 180 \mathrm{~min}$, and $240 \mathrm{~min}$ for S-1, S-2, and S-3 respectively. In Si/Au VLS mechanism, a thin $\mathrm{Au}$ layer is deposited for the wetting and formation of eutectic $\mathrm{Si} / \mathrm{Au}$ alloy phase which can rapidly adsorb Si vapor to construct one-dimensional pillars from nucleated seeds. Increasing the deposition time promotes both lateral and axial growth that leads to the formation of Si NWs with larger sizes (both cone diameter and length). It is also found that growth and annealing temperatures and Si growth rate play a significant role in the diffusion of the surface adatoms hence the morphology of Si NWs [3].

\section{Experimental Characterizations}

HR-SEM image acquisitions were first done by field emission JEOL SEM 7001F at $15-20 \mathrm{kV}$ acceleration voltages in Secondary Electron Imaging (SEI) mode. The samples were then characterized by the Grazing Angle (GI)-XRD method using Smart Lab Rigaku XRD device with $\mathrm{Cu}$ target of the wavelength of $\lambda=1.54 \AA$ at a glancing angle of $5^{\circ}$. Subsequently, energy dispersive spectroscopy (EDS) analysis and backscattered electron imaging (BEI) were performed for the elemental analysis. For enhanced fluorescence spectroscopy, the samples were drop-coated by the Rh-6G analyte, which is diluted in ethanol with a molar concentration of $10^{-9} \mathrm{~mol} / \mathrm{L}$. After the Rh-6G analyte was gently dropped on the samples, they were left to ambient temperature for drying. Finally, fluorescence spectroscopy measurements were held
Table 1 Size analyses of Aucapped Si NWs

\begin{tabular}{llll}
\hline Sample \# & Mean bottom diameter & Mean length & Number of NWs per unit area $\left(\mu \mathrm{m}^{2}\right)$ \\
\hline S-1 & $96 \pm 24$ & $264 \pm 39$ & 3.9 \\
S-2 & $138 \pm 24$ & $519 \pm 82$ & 4.0 \\
S-3 & $182 \pm 41$ & $799 \pm 122$ & 4.0 \\
\hline
\end{tabular}


by Thermo Scientific DXR Raman System with a 532-nm laser excitation at 3-mW laser output power. Semiconducting analyses were carried out with Keithley 4200-SCS Model FPP Semiconductor Analyzing System.

\section{Results and Discussions}

\section{Numerical Simulations and the Respective Optical Responses}

Different geometrical structures were previously studied via the numerical analysis using the FEM for their optical responses in the visible spectrum including the electromagnetic extinction (scattering and absorption) including $\mathrm{Ag} / \mathrm{SiO}_{2}$ and $\mathrm{Au} / \mathrm{SiO}_{2}$ core/shell NPs, $\mathrm{SiO}_{2}$-coated Ag NPs, and Ag NPs grown on $\mathrm{SiO}_{2}$ thin films [22-25]. An interesting study on the dolmen-shaped Au rectangles-capped Si thin films was demonstrated as nanoantennas in the infrared region [26]. These results demonstrate that geometrical parameters are quite important in enhancing or trapping the incident light within the plasmonic/waveguide structures. Thus, the Au-NP-capped Si NWs were designed as nanocones with different Si NWs geometrical parameters (the bottom radii and the heights of the respective nanocones) for the samples $\mathrm{S}-1, \mathrm{~S}-2$, and S-3 as schematically described in Fig. 1a. The rectangle shown in the schematic depicts the amount of Rh-6G which was preserved as constant $\left(10^{-9} \mathrm{~mol} / \mathrm{L}\right)$, and only the geometrical parameters of the Si NWs were altered for three different samples of S-1, S-2, and S-3. In S-1, the sample surface is fully covered with the Rh-6G. In S-2, only the Au-capping head without any portion from Si nanocone just appears out of Rh-6G. Furthermore, in the case of S-3, both the Au-capping head and upper side of the Si nanocone were revealed on the top of $\mathrm{Rh}-6 \mathrm{G}$.

Then, numerical simulations using the FEM were conducted for the determination of the optical responses upon the exposure of the samples to the wavelengths of $532 \mathrm{~nm}$ (the initial laser beam) and $552 \mathrm{~nm}$ (the fluorescence wavelength of Rh-6G) as shown for each case in Fig. 1b as a representation of the electric field distributions upon the excitations. It can be generalized that increasing the Si NW bottom radii and height helped to improve the extinction (primarily scattering) of the incident light. For S-1, the electric field y-component was preserved mainly by the $\mathrm{Si} \mathrm{NW}$ whereas the electric field distribution due to the fluorescence light exposure at $552 \mathrm{~nm}$ was intensified only to the region around the Au nanoparticle. For S-2, the electric field distribution has similar characteristics at the wavelength of $532 \mathrm{~nm}$ and $552 \mathrm{~nm}$ in a way that the lights extinct primarily through the Si NW body from the middle part of the Si NW cone to its bottom. Furthermore, the extinction of the incident light at $532 \mathrm{~nm}$ through S-3 occurs along the sample surface but with an emphasis at the
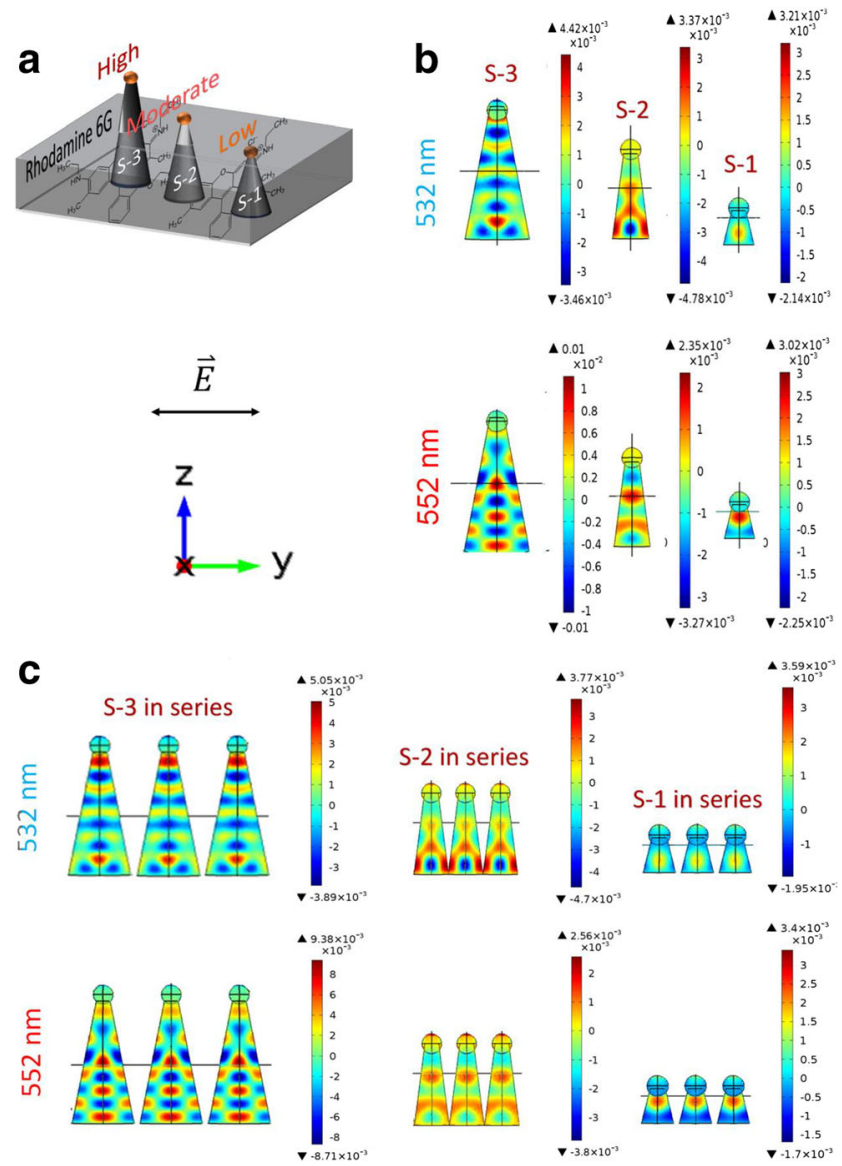

Fig. 1 a Schematic of three different samples (S-1, S-2, and S-3) were tested for the enhancement of the fluorescence of Rh-6G. It should be noted that three different geometries were only drawn together with $\mathrm{Rh}$ $6 \mathrm{G}$ for the ease of demonstration. In simulations, each sample with its own geometrical parameters is tested separately. b Results of numerical simulations performed for the electric field y-components of the single samples of S-1, S-2, and S-3 and c the same samples in series when exposed to the wavelengths of $532 \mathrm{~nm}$ and $552 \mathrm{~nm}$

bottom of the Si cone. Remarkably, S-3 also yields the extinction of the fluorescence light at $552 \mathrm{~nm}$ by a periodic electric field enhancement by a factor of 3.3 with respect to that of the S-1 from the middle of the Si nanocone to the bottom of the cone where the fluorescence light from the Rh-6G is mainly emitted.

In order to study a more realistic case in the simulation, three Au-capped Si NWs were located in series along the yaxis next to one another as the results are shown in Fig. 1c separately for each sample (S-1 in series, S-2 in series, and S-3 in series). The results demonstrate that the electric field distributions, particularly around the Au-NPs, are in general enhanced. Increasing the number of Au-capped Si NWs along the $\mathrm{y}$-axis and the size of $\mathrm{Si}$ NWs (the cone bottom diameter and the cone height) also present an enhancement of the electric dipole oriented around the Au NPs along the z-axis that was observed particularly for S-3 in series. The previous studies demonstrate the plasmonic absorption wavelength from 
$\mathrm{Au}$ NPs, and the optical band gaps from Au-doped Si are $552 \mathrm{~nm}(\cong 2.25 \mathrm{eV})[27]$ and around $605-688 \mathrm{~nm}(\cong 1.8-$ $2.05 \mathrm{eV})[28,29]$. The numerical simulation results given in this study also revealed to be consistent with the previous reports both for the plasmonic extinction wavelengths of $\mathrm{Au}$ NPs and sub-bandgap optical extinction of $\mathrm{Au} / \mathrm{Si}$ (Au-doped Si) nanostructures (Fig. S1).

\section{Morphological and Experimental Fluorescence Enhancement Studies}

In order to verify the numerical simulations experimentally, Au-capped Si NWs (S-1, S-2, and S-3) were grown using the VLS mechanism as described in the experimental section. Figure $2 \mathrm{a}, \mathrm{c}$, and e show the scanning electron microscopy (SEM) images of S-1, S-2, and S3, respectively with Rh-6G. On the other hand, Fig. 2 b, d, and f represent the SEM images of S-1, S-2, and S3 without Rh-6G. The results demonstrate in
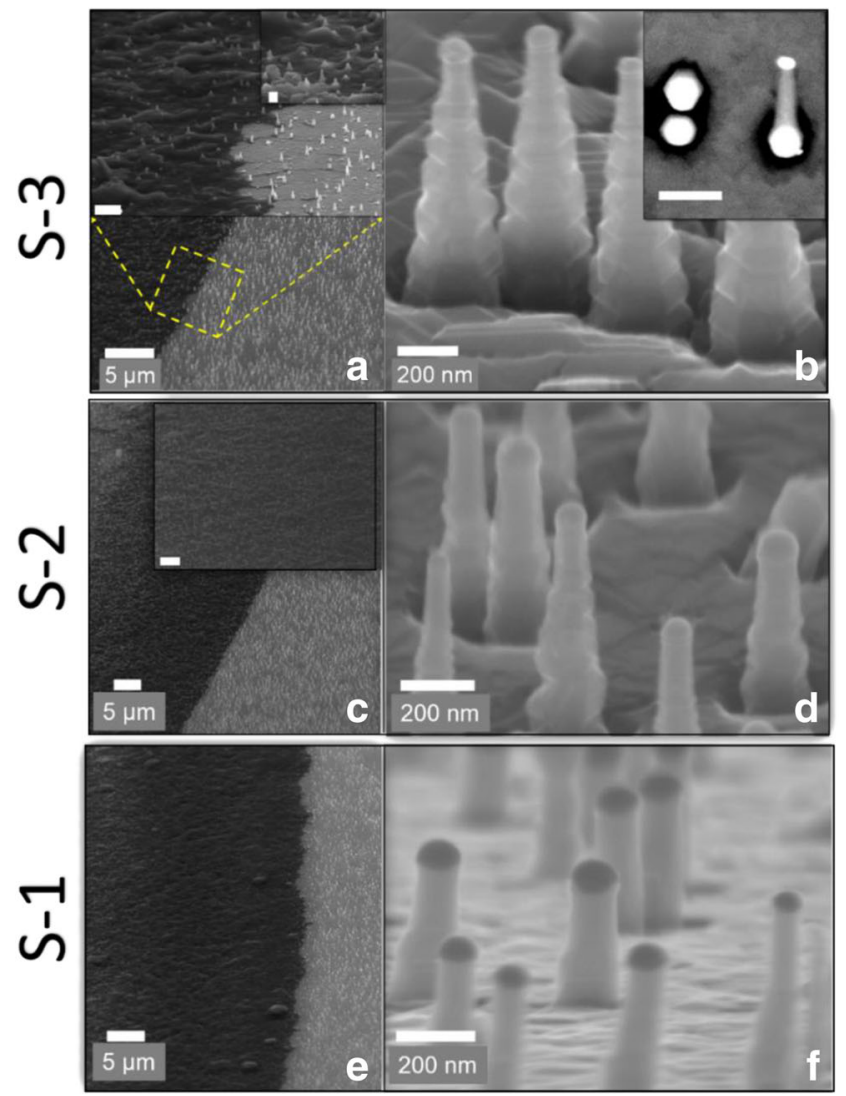

Fig. 2 SEM images of the Au-capped NWs with (black) and without (gray) the Rh-6G. a The Au-capped Si NWs in S-3 showing comparatively the largest (high) enhancement. The zoomed-in regions are shown in the insets where the scale bars are $1 \mu \mathrm{m}$ and $200 \mathrm{~nm}$. b S-3 without Rh6G. The SEM image in the backscattering mode is given in the inset where the Au-dominant part appears relatively brighter. The scale bar in the inset is $1 \mu \mathrm{m}$. $\mathbf{c}$ The Au-capped Si NWs in S-2 showing the relatively moderate enhancement. The scale bar in the inset is $1 \mu \mathrm{m}$. d S-2 without Rh-6G. e The Au-capped Si NWs in S-1 yields the lowest enhancement. f S-1 without Rh-6G
Table 1 that the geometrical parameters of the Si NWs such as the bottom radii and the lengths respectively increased from S1 to $\mathrm{S}-3$.

After close proximity to the geometrical parameters used in the numerical simulations were confirmed experimentally, Fig. 3 shows the fluorescence spectroscopy measurements of the samples S-1, S-2, and S-3 which were conducted and compared with that of Rh-6G $\left(\lambda_{\text {emis }}=552 \mathrm{~nm}\right)$ in order to verify the numerical analysis.

Au-capped Si NWs for all the samples were grown on ntype Si substrates so that the fluorescence enhancement peak was aimed not to be deteriorated due to the background Raman signal $\left(\omega(S i-S i)=520 \mathrm{~cm}^{-1}\right)$. The fluorescence enhancement factor (FEF) was found using the following equation [30]:

$\mathrm{FEF}=\frac{I_{\mathrm{NW}}-I_{0}}{I_{0}}$

where the $I_{\mathrm{NW}}$ fluorescence intensity from the Rh-6G attached next to the samples (S-1, S-2, and S-3) and $I_{0}$ is the fluorescence intensity only from the Rh-6G (without Au-capped $\mathrm{Si}$ NWs). The FEF results confirm in Table 2 that S-3 has relatively the highest fluorescence enhancement with respect to that of Rh-6G among all the samples with an average enhancement factor of 5.8 as expected from the numerical simulations. Out of these structures, the FEF results also demonstrate the optimum aspect ratio between the gold NP, and the Si NW is obtained for S-3 for which relatively smaller volume of the analyte would be sufficient. The samples with such geometry can be implemented as lab-on-chip devices as previously described [31]. Briefly, the analyte will bind to a specific biological component such as antibody and enzymes that one would like to detect, and the optical fluorescence signal would

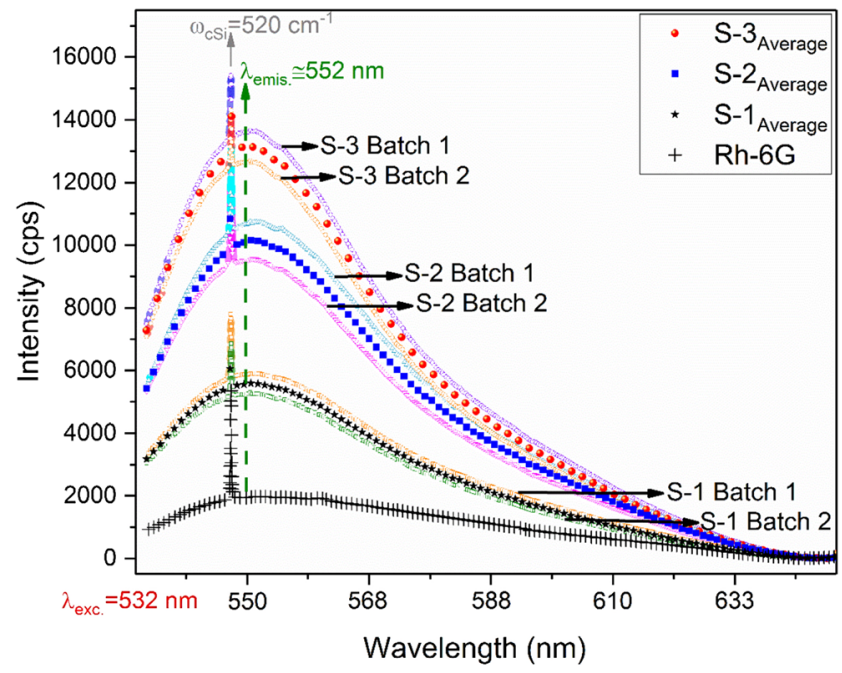

Fig. 3 The photoluminescence spectra from the different batches of the Au-capped Si NWs (S-1, S-2, and S-3) together with their average curves demonstrating yielding different fluorescence enhancement factors with respect to that of Rhodamine $6 \mathrm{G}$ on an n-type c-Si 
Table 2 The

fluorescence

(FEFs) of Au-capped Si

NWs with respect to the

$\mathrm{Rh}-6 \mathrm{G}$ as a control group enhancement factors

\begin{tabular}{lc}
\hline Sample \# & FEFs \\
\hline S-1 & 1.9 \\
S-2 & 4.2 \\
S-3 & 5.8 \\
\hline
\end{tabular}

be proportional to the amount of analyte and of the biomolecule in interest.

\section{Structural and Electrical Characterizations}

The samples were further investigated using the grazing angle XRD (GA-XRD) measurements which give information primarily from the structures of the Au-capped Si NWs due to the limitation of the X-rays for the penetration to the crystalline-Si (c-Si) substrate. Figure 4 depicts the respective crystallinity of the samples and the main diffraction peaks which were assigned as c-Si(111), $\mathrm{Au}(200)$, and $\mathrm{AuSi}(510) / \mathrm{AuSi}(431)$ (using the crystallographic information files (CIFs) available as the Crystallography Open Database (COD) card number 9008565 for cubic Si and the COD card number 9008463 for cubic Au and the National Institute for Materials Science (NIMS) identifier number 4295277376-1-2 for the AuSi alloys. In other words, the diffraction peaks at about $48^{\circ}$ are attributed to the planes (510) and (431) of AuSi alloys since they occur exactly at the same point. The Au (200) and AuSi (332) planes have reflections similarly in the same region at around $44^{\circ}$. It is well-known to use Debye-Scherrer's equation for the size estimation of the nanocrystallites and WilliamsonHall (W-H) analysis to differentiate the broadening by size

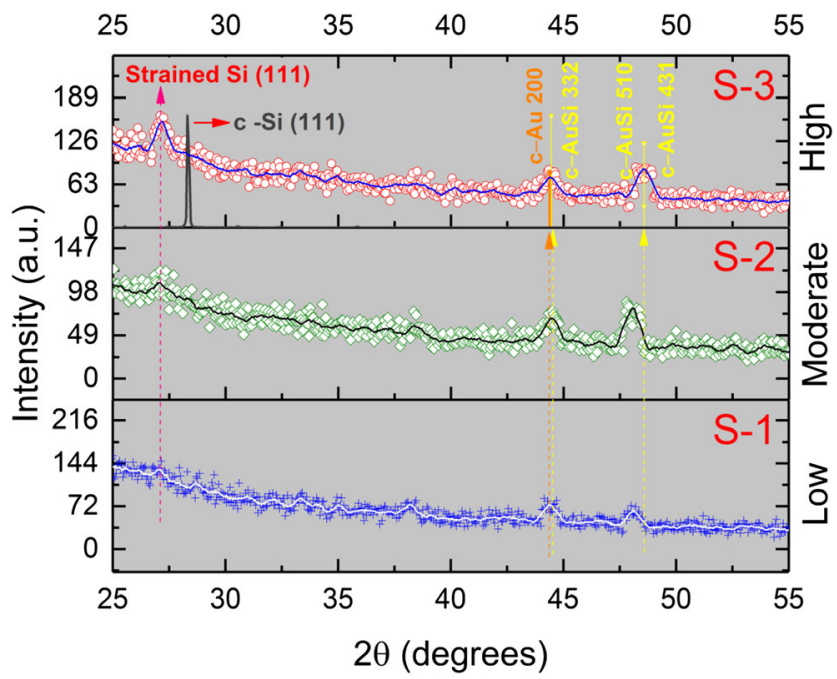

Fig. $4 \mathrm{XRD}$ data showing structural differences in the Au-capped Si NWs where the fluorescence enhancements are low, moderate, and high for the samples of S-1, S-2, and S-3 respectively. Solid lines (blue, black, and white) are drawn as a reference to the respective scattered XRD data (circle, diamond, and plus)
Table 3 Size analyses of the grain size of the nanocrystallites in the $\mathrm{Si}$ NWs using the Debye-Scherer's equation

\begin{tabular}{lllll}
\hline Sample \# & Peak position & $\begin{array}{l}\text { FWHM } \\
\text { degrees })\end{array}$ & Grain Size (nm) & $\%$ Strain in $d$ \\
\hline S-1 & - & - & - & - \\
S-2 & 27.0 & 0.9 & 9.8 & 8.1 \\
S-3 & 27.2 & 0.6 & 14.7 & 7.8 \\
\hline
\end{tabular}

and strain due to dependence on the scattering angle [32, 33]. Nevertheless, W-H analysis uses both the Young modulus $\mathrm{E}_{\mathrm{hkl}}$ of the $\mathrm{Si}$ NWs and the elastic compliance tensor $\mathrm{S}_{\mathrm{ij}}$. To the authors' knowledge, studies are limited with the polycrystalline $\mathrm{Si}$ [34] and no data available for such Si NWs with the conical or cylindrical geometries, and further studies are needed for such analysis as it is beyond the scope of the current work.
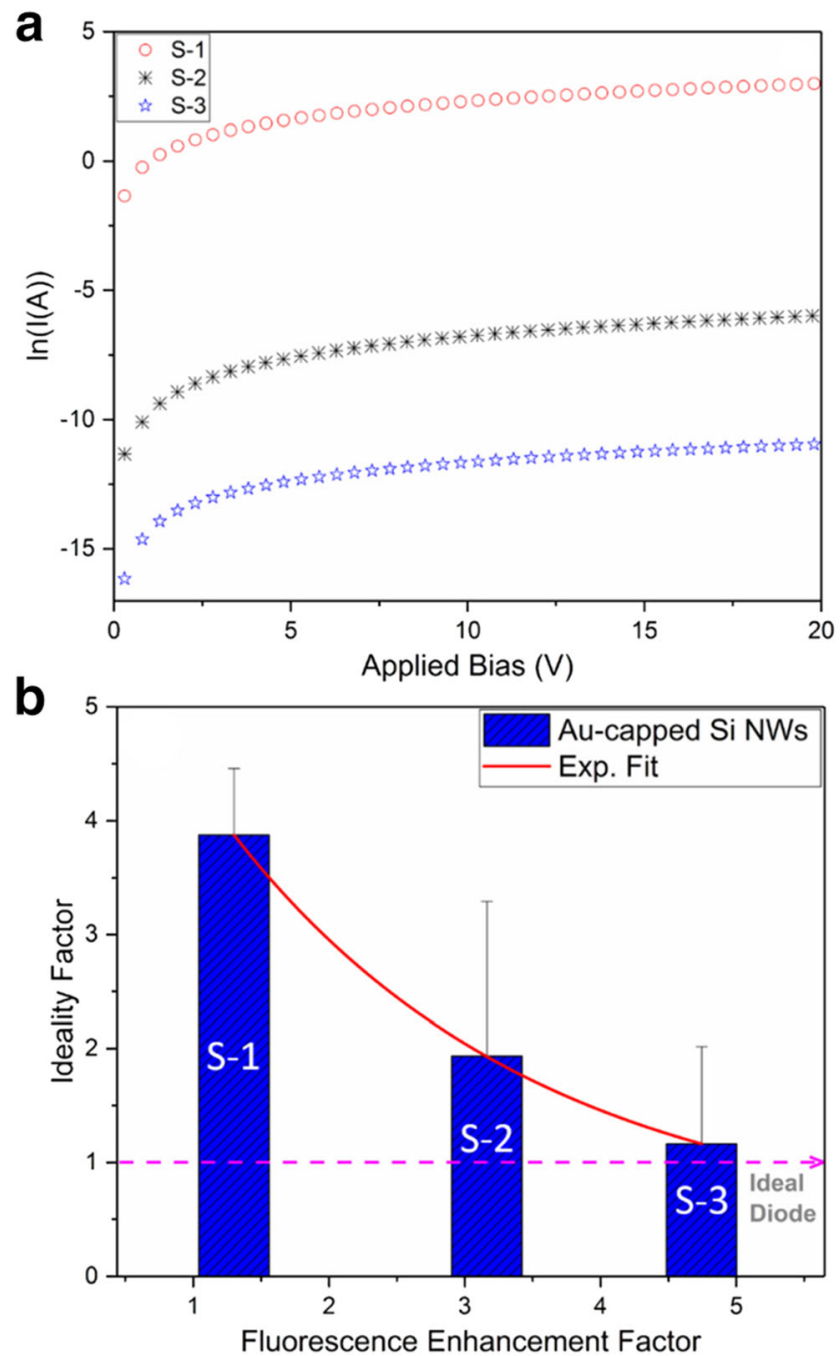

Fig. 5 a Semilogarithmic graph of I-V curve and b Schottky ideality factor vs fluorescence enhancements of Au-capped Si NWs (S-1, S-2, and S-3) 
Thus, the structure of each sample was investigated via fitting the Lorentzian functions to the c-Si(111). The fitting to the $\mathrm{c}-\mathrm{Si}(111)$ peak for the S-1 sample was not performed due to the fact that the peak was extremely weak. Table 3 demonstrates the corresponding crystallite sizes found from the Debye-Scherrer's equation and the percentages of the strain in the interlayer distance $d$ with respect to the bulk c$\mathrm{Si}(d=3.1353 \AA)$ where the $d$ values for the S- 2 and S- 3 were calculated using the well-known Bragg's equation. Table S1 gives the elemental analyses of the samples and found to be close to one another as the same Au growth rate was utilized for the Au-caps.

Upon investigating the structures of Au-capped Si NWs, the electrical analysis was performed using I-V (four-point probe) measurements as demonstrated in Fig. 5.

The ideality factor was determined from the slope of the linear region of the $\ln I-\mathrm{V}$ plot given in Fig. $5 \mathbf{a}$ at forward bias using the Cheung's equation [35] as follows:

$\frac{d V}{d \ln I}=n k T / q$

where $k$ is the Boltzmann constant, $n$ is the ideality factor, $T$ is the temperature of the ambient environment, and $q$ is the electrical charge of the current carriers. Figure $5 \mathrm{~b}$ demonstrates that the Schottky ideality factor decays exponentially when the fluorescence enhancements of Au-capped Si NWs were improved from S-1 to S-3. In other words, the ideality parameter of S-3 approaches to unity close to an ideal diode for which a relatively higher enhancement was detected.

\section{Conclusion}

In summary, the fluorescence enhancement factors of the Aucapped Si NWs were determined at various sizes with respect to the fluorescence emission from $\mathrm{Rh}-6 \mathrm{G}$ fluorophore. Both numerical and experimental results verify that when the size between the fluorophore and the Au-NP capping is highest, the maximum fluorescence enhancement was recorded. It is also found that the Schottky ideality factor of Au-capped Si NWs decays exponentially when improving the fluorescence enhancements.

Acknowledgments The authors acknowledge the support provided by Bilkent University UNAM.

Authors Contributions The works in the project were coordinated by Dr. İsa Șeker. The manuscript was written by Dr. İsa Șeker and Dr. Ali Karatutlu. The numerical analyses were performed by Dr. Ali Karatutlu and Dr. Bülend Ortaç. Fabrication of Au-capped Si NWs were conducted by Dr. İsa Șeker and Dr. Mehmet Karakız. Fluorescence enhancement studies of Rh-6G were conducted and recorded by Dr. İsa Șeker and Dr. Kurtuluș Gölcük. Dr. Ali Karatutlu and Dr. İsa Șeker also performed the morphological, structural, and electrical analyses of Au-capped Si NWs.

\section{Compliance with Ethical Standards}

Conflicts of Interest The authors declare that they have no conflict interest.

\section{References}

1. Ayala-Orozco C, Liu JG, Knight MW, Wang Y, Day JK, Nordlander P, Halas NJ (2014) Fluorescence enhancement of molecules inside a gold nanomatryoshka. Nano Lett 14:2926-2933. https://doi.org/10.1021/n1501027j

2. Holmes JD, Johnston KP, Doty RC, Korgel BA (2000) Control of thickness and orientation of solution-grown silicon nanowires. Science 287:1471-1473. https://doi.org/10.1126/science.287. 5457.1471

3. Karakiz M, Toydemir B, Unal B, Colakerol Arslan L (2014) Growth of shape controlled silicon nanowhiskers by electron beam evaporation. The European Physical Journal Applied Physics 65: 20403. https://doi.org/10.1051/epjap/2014130362

4. Sokolov LV, Gavrilova TA, Werner P et al (2008) Molecular-beam epitaxy-grown $\mathrm{Si}$ whisker structures: morphological, optical and electrical properties. Nanotechnology 19:225708. https://doi.org/ 10.1088/0957-4484/19/22/225708

5. Schmidt MS, Hübner J, Boisen A (2012) Large area fabrication of leaning silicon nanopillars for surface enhanced Raman spectroscopy. Adv Mater 24:11-18. https://doi.org/10.1002/adma.201103496

6. Oldenburg SJ, Averitt RD, Westcott SL, Halas NJ (1998) Nanoengineering of optical resonances. Chem Phys Lett 288: 243-247. https://doi.org/10.1016/S0009-2614(98)00277-2

7. Abadeer NS, Brennan MR, Wilson WL, Murphy CJ (2014) Distance and plasmon wavelength dependent fluorescence of molecules bound to silica-coated gold nanorods. ACS Nano 8:83928406. https://doi.org/10.1021/nn502887j

8. Wu Y, Yang P (2001) Direct observation of vapor-liquid-solid nanowire growth. J Am Chem Soc 123:3165-3166. https://doi. org/10.1021/ja0059084

9. Ge S, Jiang K, Lu X, Chen Y, Wang R, Fan S (2005) Orientationcontrolled growth of single-crystal silicon-nanowire arrays. Adv Mater 17:56-61. https://doi.org/10.1002/adma.200400474

10. Wu Y, Cui Y, Huynh L, Barrelet CJ, Bell DC, Lieber CM (2004) Controlled growth and structures of molecular-scale silicon nanowires. Nano Lett 4:433-436. https://doi.org/10.1021/n1035162i

11. Yan H, Xing Y, Hang Q et al (2000) Growth of amorphous silicon nanowires via a solid-liquid-solid mechanism. Chem Phys Lett 323:224-228. https://doi.org/10.1016/S0009-2614(00)00519-4

12. Trentler TJ, Hickman KM, Goel SC, Viano AM, Gibbons PC, Buhro WE (1995) Solution-liquid-solid growth of crystalline IIIV semiconductors: an analogy to vapor-liquid-solid growth. Science 270:1791-1794. https://doi.org/10.1126/science.270. 5243.1791

13. Chen SY, Chen LJ (2006) Self-assembled epitaxial NiSi2 nanowires on $\mathrm{Si}(001)$ by reactive deposition epitaxy. Thin Solid Films 508:222-225. https://doi.org/10.1016/j.tsf.2005.07.322

14. Wang Y, Schmidt V, Senz S, Gösele U (2006) Epitaxial growth of silicon nanowires using an aluminium catalyst. Nat Nanotechnol 1: 186-189. https://doi.org/10.1038/nnano.2006.133

15. Zhang R-Q, Lifshitz Y, Lee S-T (2003) Oxide-assisted growth of semiconducting nanowires. Adv Mater 15:635-640. https://doi.org/ 10.1002/adma.200301641

16. Yu DP, Lee CS, Bello I, Sun XS, Tang YH, Zhou GW, Bai ZG, Zhang Z, Feng SQ (1998) Synthesis of nano-scale silicon wires by excimer laser ablation at high temperature. Solid State Commun 105:403-407. https://doi.org/10.1016/S0038-1098(97)10143-0 
17. Peng KQ, Hu JJ, Yan YJ, Wu Y, Fang H, Xu Y, Lee ST, Zhu J (2006) Fabrication of single-crystalline silicon nanowires by scratching a silicon surface with catalytic metal particles. Adv Funct Mater 16:387-394. https://doi.org/10.1002/adfm.200500392

18. Chakraborti S, Basu RN, Panda SK (2018) Vertically aligned silicon nanowire array decorated by Ag or Au nanoparticles as SERS substrate for bio-molecular detection. Plasmonics 13:1057-1080. https://doi.org/10.1007/s11468-017-0605-2

19. Zhu J, Yu Z, Burkhard GF, Hsu CM, Connor ST, Xu Y, Wang Q, McGehee M, Fan S, Cui Y (2009) Optical absorption enhancement in amorphous silicon nanowire and nanocone arrays. Nano Lett 9: 279-282. https://doi.org/10.1021/n1802886y

20. Attaran A, Emami SD, Soltanian MRK, Penny R, behbahani F, Harun SW, Ahmad H, Abdul-Rashid HA, Moghavvemi M (2014) Circuit model of Fano resonance on tetramers, pentamers, and broken symmetry Pentamers. Plasmonics 9:1303-1313. https://doi. org/10.1007/s11468-014-9743-y

21. Stöger-Pollach M, Bukvišová K, Schwarz S, Kvapil M, Šamořil T, Horák M (2019) Fundamentals of cathodoluminescence in a STEM: the impact of sample geometry and electron beam energy on light emission of semiconductors. Ultramicroscopy 200:111124. https://doi.org/10.1016/j.ultramic.2019.03.001

22. Ge W, Zhang X, Liu M, et al (2014) Plasmonic properties of Au/ $\mathrm{SiO} 2$ nanoparticles: effect of gold size and silica dielectric layer thickness. Materials Research Innovations 18:S4-701-S4-705. https://doi.org/10.1179/1432891714Z.000000000769

23. Sharma R, Roopak S, Kumar PN et al (2017) Study of surface plasmon resonances of core-shell nanosphere: a comparison between numerical and analytical approach. Plasmonics 12:977986. https://doi.org/10.1007/s11468-016-0349-4

24. Nomura K-I, Fujii S, Ohki Y, Awazu K, Fujimaki M, Tominaga J, Fukuda N, Hirakawa T, Rockstuhl C (2008) Fabrication of inert silver nanoparticles with a thin silica coating. Jpn J Appl Phys 47: 8641-8643. https://doi.org/10.1143/JJAP.47.8641

25. Mandal A, Chaudhuri P (2013) Controlling the absorption spectrum within a thin amorphous silicon layer by using the size dependent plasmonic behaviour of silver nanoparticles. Journal of Renewable and Sustainable Energy 5:031614. https://doi.org/10.1063/1. 4809787
26. Vyas H, Hegde RS (2019) Waveguide interrogation of a compound plasmonic nanoantenna. Journal of Nanophotonics 13:1. https://doi. org/10.1117/1.JNP.13.026004

27. Taheri M, Hajiesmaeilbaigi F, Motamedi AS, Golian Y (2015) Nonlinear optical response of gold/silicon nanocomposite prepared by consecutive laser ablation. Laser Physics 25:. https://doi.org/10. 1088/1054-660X/25/6/065901, 25

28. Ferdous N, Ertekin E (2018) Atomic scale origins of sub-band gap optical absorption in gold-hyperdoped silicon. AIP Adv 8:55014. https://doi.org/10.1063/1.5023110

29. Ghosh R, Imakita K, Fujii M, Giri PK (2016) Effect of Ag/Au bilayer assisted etching on the strongly enhanced photoluminescence and visible light photocatalysis by Si nanowire arrays. Phys Chem Chem Phys 18:7715-7727. https://doi.org/10. 1039/C5CP07161E

30. Mei Z, Tang L (2017) Surface-plasmon-coupled fluorescence enhancement based on ordered gold nanorod array biochip for ultrasensitive DNA analysis. Anal Chem 89:633-639. https://doi.org/ 10.1021/acs.analchem.6b02797

31. Drake C, Deshpande S, Bera D, Seal S (2007) Metallic nanostructured materials based sensors. Int Mater Rev 52:289-317. https:// doi.org/10.1179/174328007X212481

32. Williamson G, Hall W (1953) X-ray line broadening from filed aluminium and wolfram. Acta Metall 1:22-31. https://doi.org/10. 1016/0001-6160(53)90006-6

33. Mote V, Purushotham Y, Dole B (2012) Williamson-Hall analysis in estimation of lattice strain in nanometer-sized $\mathrm{ZnO}$ particles. Journal of Theoretical and Applied Physics 6:6. https://doi.org/10. 1186/2251-7235-6-6

34. Cantwell PR, Kim H, Schneider MM, Hsu HH, Peroulis D, Stach EA, Strachan A (2012) Estimating the in-plane Young's modulus of polycrystalline films in MEMS. J Microelectromech Syst 21: 840-849. https://doi.org/10.1109/JMEMS.2012.2191939

35. Cheung SK, Cheung NW (1986) Extraction of Schottky diode parameters from forward current-voltage characteristics. Appl Phys Lett 49:85-87. https://doi.org/10.1063/1.97359

Publisher's Note Springer Nature remains neutral with regard to jurisdictional claims in published maps and institutional affiliations. 